\title{
Cannabinoids for the Treatment of Chronic Pain: A Critical Review of Randomized Controlled Trials
}

Samah Hassan*

Institute of Medical Sciences, University of Toronto, Toronto, ON M5S, Canada

\begin{abstract}
Persistent or Chronic pain (CP) continues to be one of the most challenging health problems in Canada. CP is estimated to affect around $29 \%$ of the Canadian population. The impact of CP is enormous, as it leads to a severe decline in the quality of life as well as a startling rise in the incidence of disability. Many approaches including pharmacological, physical and psychological have been proposed for CP treatment. Yet these options have always been associated with either significant side effects or insignificant efficacy on the long run. Notably, many patients, suffering from CP conditions, are now using cannabinoids, even without a prescription. As a result, an exceeding number of patients are making extraordinary claims about how using marijuana have alleviated their pain. But the medical field thus far has not reached the same level of certainty. This situation has framed compelling reasons to explore the true effectiveness of cannabinoids for CP. The purpose of this paper is to critically review methodological quality and outcome measures used by RCTs investigating the effectiveness of cannabinoids to determine the real effect in such trials.
\end{abstract}

Keywords: Cannabiniods; Chronic pain; Quality of life

\section{Introduction}

Persistent or Chronic pain (CP) continues to be one of the most challenging health problems in Canada [1]. CP is estimated to affect around $29 \%$ of the Canadian population and accounts for up to $78 \%$ of clinical visits [2-4]. The impact of CP is enormous, as it leads to a severe decline in the quality of life as well as a startling rise in the incidence of disability. $\mathrm{CP}$ is not only associated with serious health consequences, but it also poses serious societal implications [5,6]. In 2010, the Chronic Pain Association of Canada reported that "...the annual cost of chronic pain, including medical expenses, lost income, and lost productivity is estimated to exceed $\$ 10$ billion" [6]. Many approaches including pharmacological, physical and psychological have been proposed for $\mathrm{CP}$ treatment. Yet these options have always been associated with either significant side effects or insignificant efficacy on the long run [7].

Cannabinoids have been used in one form or another for pain treatment for a long time [8]. Notably, many patients, suffering from CP conditions, are now using cannabinoids, even without a prescription. As a result, an exceeding number of patients are making extraordinary claims about how using marijuana have alleviated their pain and turned their lives around for the better [9]. But the medical field thus far has not reached the same level of certainty. In many cases, health care providers are reluctant to support the claimed outstanding benefits for cannabinoids.

This situation has framed compelling reasons to explore the true effectiveness of cannabinoids for CP. Moreover, the increasing number of patients using cannabinoids highlights the urgent need to provide solid evidence. This evidence is needed either to support or prevent such treatment before we witness another epidemic that we cannot control, similar to what we now face with opioids [10].

\section{So what does the scientific literature say?}

Since randomized controlled trials (RCTs) are considered as the only reliable method for establishing evidence for effective therapy [11], the purpose of this paper is to critically review methodological quality and outcome measures used by RCTs investigating the effectiveness of cannabinoids to determine the real effect in such trials. First, I will review published RCTs. Second, I will appraise the methods utilized.
Then, with this background, I will discuss the reliability of their conclusions concerning the efficacy that was tested and its implications for theory, research and practice.

\section{Methods}

The review was performed according to the Preferred Reporting Items for Systematic Reviews and Meta-Analyses (PRISMA) statement.

\section{Inclusion criteria}

Types of studies: Only RCTs comparing a cannabinoid with a placebo or active control group were included. An RCT is defined as "a study design that randomly assigns participants into an experimental group or a control group. As the study is conducted, the only expected difference between the control and experimental groups in an RCT is the outcome variable being studied".

\section{Types of patients}

Studies dealing with adults (18 and more years of age) who have $\mathrm{CP}$, defined as "Persistent pain, lasting for more than 3 months beyond time needed for tissue healing" [12]. CP is usually presented as an assorted set of conditions that includes chronic back pain, chronic headaches, temporomandibular disorder, fibromyalgia, myofascial pain, neuropathic pain, HIV neuropathy, rheumatoid arthritis, and osteoarthritis.

\section{Types of interventions}

Cannabinoids (such as plant-based cannabinoids (Nabiximol) or synthetic cannabinoids (e.g., cannabidiol, dronabinol, nabilone) at

${ }^{*}$ Corresponding author: Samah Hassan, Institute of Medical Sciences, University of Toronto, Toronto, ON M5S, Canada, Tel: 16474698447, E-mail: sam.hassan@mail.utoronto.ca

Received January 25, 2018; Accepted February 16, 2018; Published February 23, 2018

Citation: Hassan S (2018) Cannabinoids for the Treatment of Chronic Pain: A Critical Review of Randomized Controlled Trials. J Pain Manage Med 4: 131.

Copyright: (C) 2018 Hassan S. This is an open-access article distributed under the terms of the Creative Commons Attribution License, which permits unrestricted use, distribution, and reproduction in any medium, provided the original author and source are credited. 
Citation: Hassan S (2018) Cannabinoids for the Treatment of Chronic Pain: A Critical Review of Randomized Controlled Trials. J Pain Manage Med 4: 131.

any dose, by any route, administered for the relief of $\mathrm{CP}$, compared to placebo or any active comparators were included.

\section{Types of outcome measures}

Outcomes were selected based on the list suggested by The Initiative on Methods, Measurement, and Pain Assessment in Clinical Trials (IMMPACT) [13]. The review groups have developed a set of six domains that should be assessed in RCTs investigating the efficacy of any treatment compared to another or to placebo (Table 1).

\begin{tabular}{|c|c|}
\hline Outcome & Tool \\
\hline Pain & $\begin{array}{l}\text { - } \begin{array}{l}\text { 11-point }(0-10) \text { numerical rating scale of pain } \\
\text { intensity }\end{array} \\
\text { - } \quad \begin{array}{l}\text { Usage of rescue analgesics } \\
\text { Categorical rating of pain intensity (none, mild, } \\
\text { moderate, severe) in circumstances in which } \\
\text { numerical ratings may be problematic }\end{array}\end{array}$ \\
\hline Physical functioning & 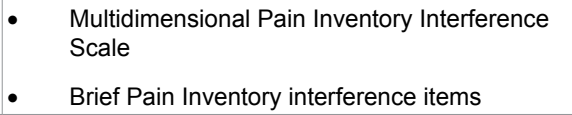 \\
\hline Emotional functioning & $\begin{array}{l}\text { Beck Depression Inventory Profile of Mood } \\
\text { States }\end{array}$ \\
\hline $\begin{array}{l}\text { Global improvement } \\
\text { and satisfaction with } \\
\text { treatment }\end{array}$ & - $\quad$ Patient Global Impression of Change \\
\hline $\begin{array}{l}\text { Symptoms and adverse } \\
\text { events }\end{array}$ & $\begin{array}{l}\text { - Passive capture of spontaneously reported } \\
\text { adverse events and symptoms and use of } \\
\text { open-ended prompts }\end{array}$ \\
\hline Participant disposition & $\begin{array}{l}\text { Detailed information regarding participant } \\
\text { recruitment and progress through the trial, } \\
\text { including all information specified in the } \\
\text { CONSORT guidelines. }\end{array}$ \\
\hline
\end{tabular}

Table 1: Recommended core outcome measures for clinical trials of chronic pain treatment efficacy and effectiveness.

\section{Search strategy}

An online search was performed in MEDLINE (via Ovid) and The Cochrane Library (via Wiley) database for studies published in the English language until $15^{\text {th }}$ March 2017. Search terms used were included in full (Appendix A). The reference lists of all identified papers were searched for additional potentially relevant publications.

All articles were screened using the inclusion criteria. If the criteria were met, the full text was then reviewed for eligibility. Only full publications were selected, while abstracts, nonrandomized, studies of experimental pain, case reports, and clinical observations were all excluded.

\section{Methodological quality assessment}

Selected RCTs were assessed for scientific quality using a validated quality instrument; the Consolidated Standards of Reporting Trials (CONSORT) 2010. The consort statement comprises a 25-item checklist. The consort tool is developed to enable readers to understand a trial's design, conduct, analysis and interpretation, and to assess the validity of its results.

\section{Assessment of risk of bias}

To assess the risk of bias, the Jadad scale was used [14]. This instrument is used to measure the likelihood of bias especially in pain research and to evaluate the impact of blinding on the findings. The instrument is simple, short, reliable, and valid. It contains three items (Randomization, Double Blinding, and Withdrawals \& Dropouts). Items are rated based on the quality of the description of the methods utilized in randomization and/or on the quality of the description of the method employed in blinding.

\section{Results}

Five-hundred and twenty-six potentially eligible articles were

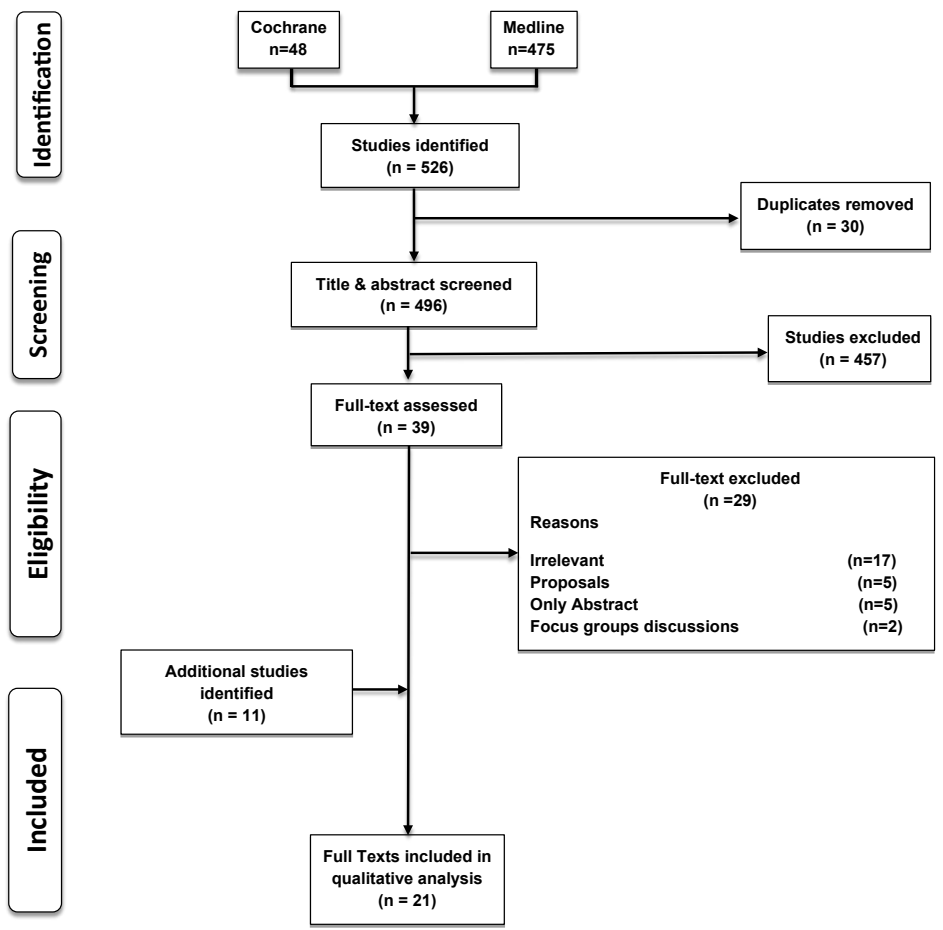

Figure 1: PRISMA (Preferred Reporting Items for Systematic Reviews and Meta-Analyses) flow chart for identified search. 
Citation: Hassan S (2018) Cannabinoids for the Treatment of Chronic Pain: A Critical Review of Randomized Controlled Trials. J Pain Manage Med 4: 131.

found from the search strategies and 11 other potential articles through review of the references. Fifty relevant studies were subjected to fulltext review (Figure 1). Altogether, this review identified 21 RCTs.

\section{Assessment of trials quality according to CONSORT}

\section{Study characteristics}

All selected trials were identified as RCTs in the title, with a structured abstract for methods, results and conclusions included. All trials provided a scientific background and explained briefly the reason behind the trials. They all determined the outcomes whether primary or secondary and proposed that cannabinoids might show more pain relief compared to placebo or the compared drug. All 21 trials were conducted from 2003 to 2014, investigating cannabinoids in different forms with a mean duration of treatment of 3.4 weeks (Table 2).

13 of the 21 trials were in neuropathic pain [15-24], another in chronic motor neuron syndrome and one in chronic headaches $[25,26]$.

\section{Methods}

Trial design: All 21 studies were double-blinded, 15 used a crossover design [27-30].

In parallel RCTs, the baseline for patients assigned to one group may have significant differences from those allocated to the other group. Hence, it is very important to routinely collect baseline data before treatment begins. Nevertheless, Toth and his colleagues did not report any baseline measurements in their trial.

\section{Participants}

The 21 trials involved a total of 1614 participants that met the inclusion criteria. The inclusion criteria for recruiting patients for all trials were mainly patients diagnosed with the pain condition under investigation, age 18 years and older.

A significant part of any study design is the standardized definition and diagnosis criteria of the entity under investigation, one of the

\begin{tabular}{|c|c|c|c|c|c|c|c|c|c|c|}
\hline$\#$ & Study & Design & $\begin{array}{c}\text { Type of Chronic } \\
\text { Pain }\end{array}$ & \multicolumn{2}{|c|}{$\begin{array}{l}\text { Study Sample } \\
\text { Exp. / Control }\end{array}$} & \multirow{2}{*}{\begin{tabular}{|c|} 
Intervention \\
Oromucosal \\
spray $2.7 \mathrm{mg} \mathrm{THC}$ \\
$\& 2.5 \mathrm{CBD}$ vs \\
Placebo spray
\end{tabular}} & \multirow{2}{*}{$\begin{array}{l}\text { Duration } \\
5 \text { weeks }\end{array}$} & \multirow{2}{*}{$\begin{array}{c}\text { Outcome } \\
\text { Pain } \\
\text { Sleep }\end{array}$} & \multirow{2}{*}{\begin{tabular}{|c|}
\multicolumn{1}{|c}{ Results } \\
$\begin{array}{l}\text { Significant decrease } \\
\text { in pain intensity and } \\
\text { improved sleep }\end{array}$ \\
\end{tabular}} & \multirow{2}{*}{$\begin{array}{c}\begin{array}{c}\text { Side effects \& } \\
\text { Disposition }\end{array} \\
\text { Not mentioned }\end{array}$} \\
\hline 1 & $\begin{array}{l}\text { Blake et } \\
\text { al.[2] }\end{array}$ & Parallel & $\begin{array}{l}\text { Rheumatoid } \\
\text { arthritis }\end{array}$ & 29 & 29 & & & & & \\
\hline 2 & $\begin{array}{l}\text { Skrabek } \\
\text { et al.[5] }\end{array}$ & Parallel & Fibromyalgia & 20 & 20 & $\begin{array}{l}\text { Nabilone } 1 \mathrm{mg} / \mathrm{d} \\
\text { orally vs Placebo }\end{array}$ & 4 weeks & $\begin{array}{c}\text { Pain (VAS) } \\
\text { Anxiety } \\
\text { HRQL }\end{array}$ & $\begin{array}{l}\text { Significant reduction } \\
\text { in pain, anxiety and } \\
\text { in quality of life }\end{array}$ & $\begin{array}{l}7 \text { withdrawals due } \\
\text { to dry mouth, \& } \\
\text { drowsiness }\end{array}$ \\
\hline 3 & $\begin{array}{l}\text { Ware et } \\
\text { al.[6] }\end{array}$ & Crossover & Fibromyalgia & 21 & 23 & $\begin{array}{c}\text { Nabilone } \\
\text { 1mg/d orally Vs } \\
\text { Amitriptylline 10- } \\
20 \mathrm{mg}\end{array}$ & 2 weeks & $\begin{array}{c}\text { Pain (NRS, } \\
\text { McGill } \\
\text { questionnaire) } \\
\text { Sleep } \\
\text { HRQL }\end{array}$ & No difference & $\begin{array}{l}3 \text { withdrawals Due to } \\
\text { side effects: sedation } \\
\text { \& dry mouth and loss } \\
\text { of effectiveness }\end{array}$ \\
\hline 4 & $\begin{array}{c}\text { Notcutt et } \\
\text { al.[39] }\end{array}$ & Crossover & Chronic pain & 34 & 34 & $\begin{array}{c}\text { Sublingual THC } \\
\text { vs cannabidol vs } \\
\text { both combo vs } \\
\text { placebo }\end{array}$ & 12 weeks & $\begin{array}{l}\text { Pain } \\
\text { Sleep } \\
\text { BDI }\end{array}$ & $\begin{array}{l}\text { THC \& both combo } \\
\text { were superior to } \\
\text { placebo }\end{array}$ & $\begin{array}{l}5 \text { withdrawals Nausea } \\
\text { vomiting depression }\end{array}$ \\
\hline 5 & $\begin{array}{c}\text { Pinsger et } \\
\text { al.[27] }\end{array}$ & Crossover & Chronic pain & 30 & 30 & $\begin{array}{l}\text { Nabilone } 0.25- \\
1 \mathrm{mg} / \mathrm{d} \text { orally vs } \\
\text { Placebo }\end{array}$ & 16 weeks & $\begin{array}{c}\text { Pain (VAS) } \\
\text { HRQL }\end{array}$ & $\begin{array}{l}\text { Significant reduction } \\
\text { in pain and } \\
\text { improvement in } \\
\text { quality of life }\end{array}$ & Not mentioned \\
\hline 6 & $\begin{array}{c}\text { Narang et } \\
\text { al.[38] }\end{array}$ & Crossover & Chronic pain & 29 & 30 & $\begin{array}{l}\text { Dronabinol } 20 \mathrm{mg} \\
\text { orally vs placebo }\end{array}$ & 4 weeks & $\begin{array}{l}\text { Pain (VAS, } \\
\text { BPI) HADS } \\
\text { Sleep }\end{array}$ & Significant pain relief & $\begin{array}{l}3 \text { dropped out Dry } \\
\text { mouth, dizziness }\end{array}$ \\
\hline \multirow[t]{2}{*}{7} & \multirow{2}{*}{$\begin{array}{l}\text { Pini et } \\
\text { al.[4] }\end{array}$} & \multirow[t]{2}{*}{ Crossover } & \multirow{2}{*}{$\begin{array}{c}\text { Chronic } \\
\text { Headaches }\end{array}$} & \multirow[t]{2}{*}{26} & \multirow[t]{2}{*}{30} & \multirow{2}{*}{$\begin{array}{c}\text { Nabilone } 0.5 \mathrm{mg} / \\
\text { day vs Ibuprofen } \\
400 \mathrm{mg} / \mathrm{day}\end{array}$} & \multirow[t]{2}{*}{2 weeks } & Pain (VAS) & $\begin{array}{l}\text { Nabilone was } \\
\text { superior to ibuprofen } \\
\text { in reducing pain } \\
\text { intensity }\end{array}$ & \multirow{2}{*}{$\begin{array}{c}4 \text { dropped out for lack } \\
\text { of efficacy }\end{array}$} \\
\hline & & & & & & & & $\begin{array}{l}\text { QOL Mood, } \\
\text { depression \& } \\
\text { anxiety }\end{array}$ & $\begin{array}{l}\text { Both drugs showed } \\
\text { improvement in all } \\
\text { other variables }\end{array}$ & \\
\hline 8 & $\begin{array}{c}\text { Wissel et } \\
\text { al.[8] }\end{array}$ & Crossover & $\begin{array}{l}\text { Chronic upper } \\
\text { motor neuron } \\
\text { Syndrome }\end{array}$ & 13 & 13 & $\begin{array}{c}\text { Nabilone } 1 \mathrm{mg} \\
\text { orally vs Placebo }\end{array}$ & 1 week & $\begin{array}{l}\text { Pain (11-piont } \\
\text { text box) }\end{array}$ & $\begin{array}{l}\text { Significant decrease } \\
\text { in pain ratings }\end{array}$ & $\begin{array}{c}2 \text { withdrawals } \\
\text { weakness in LL } \\
\text { Dizziness, Vertigo, } \\
\text { drowsiness }\end{array}$ \\
\hline \multirow[t]{2}{*}{9} & \multirow{2}{*}{$\begin{array}{l}\text { Ellis et } \\
\text { al.[34] }\end{array}$} & \multirow[t]{2}{*}{ Crossover } & \multirow[t]{2}{*}{ HIV-Neuropathy } & \multirow[t]{2}{*}{34} & \multirow[t]{2}{*}{34} & \multirow{2}{*}{$\begin{array}{l}\text { Cannabis ( } 1 \%- \\
8 \% \text { THC) smoked } \\
\text { vs Placebo }\end{array}$} & \multirow[t]{2}{*}{5 days } & \multirow{2}{*}{$\begin{array}{c}\text { Pain (Vas } \\
\& \text { McGill } \\
\text { questionnaire) }\end{array}$} & \multirow{2}{*}{$\begin{array}{l}\text { Significant reduction } \\
\text { in pain }\end{array}$} & $\begin{array}{c}6 \text { withdrawals } \\
\text { Concentration } \\
\text { difficulties, fatigue, } \\
\text { sleepiness or sedation, }\end{array}$ \\
\hline & & & & & & & & & & $\begin{array}{l}\text { Increased duration } \\
\text { of sleep, reduced } \\
\text { salivation, and thirst. }\end{array}$ \\
\hline 10 & $\begin{array}{c}\text { Abrams et } \\
\text { al.[15] }\end{array}$ & Parallel & HIV-Neuropathy & 27 & 28 & $\begin{array}{c}\text { Cannabis }(3.56 \% \\
\text { THC) smoked vs } \\
\text { Placebo }\end{array}$ & 5 days & Pain (VAS) & $\begin{array}{l}\text { Significant decrease } \\
\text { in pain }\end{array}$ & $\begin{array}{l}\text { No withdrawals No } \\
\text { side effects }\end{array}$ \\
\hline 11 & $\begin{array}{l}\text { Wilsey et } \\
\text { al.[22] }\end{array}$ & Crossover & Neuropathic pain & 38 & 38 & $\begin{array}{l}\text { Cannabis }(7 \% \\
\text { THC) smoked vs } \\
\text { Placebo }\end{array}$ & 21 days & Pain (VAS) & $\begin{array}{l}\text { Significant reduction } \\
\text { in pain }\end{array}$ & $\begin{array}{c}6 \text { withdrawals Some } \\
\text { acute cognitive effects, } \\
\text { particularly with } \\
\text { memory, at higher } \\
\text { doses }\end{array}$ \\
\hline
\end{tabular}


Citation: Hassan S (2018) Cannabinoids for the Treatment of Chronic Pain: A Critical Review of Randomized Controlled Trials. J Pain Manage Med 4: 131.

Page 4 of 7

\begin{tabular}{|c|c|c|c|c|c|c|c|c|c|c|}
\hline 12 & $\begin{array}{l}\text { Wilsey et } \\
\text { al.[21] }\end{array}$ & Crossover & Neuropathic pain & 38 & 44 & $\begin{array}{c}\text { Cannabis }(7.7 \%) \\
\text { smoked vs } \\
\text { placebo }\end{array}$ & 14 days & $\begin{array}{l}\text { Pain (VAS) } \\
\text { PGIC }\end{array}$ & $\begin{array}{l}\text { Significant } \\
\text { reduction in pain } \\
\& \text { improvement in } \\
\text { global impression of } \\
\text { change scores }\end{array}$ & No withdrawals \\
\hline 13 & $\begin{array}{l}\text { Ware et } \\
\text { al.[32] }\end{array}$ & Crossover & Neuropathic pain & 23 & 23 & $\begin{array}{l}\text { THC (6\%) } \\
25 \mathrm{mg} / \mathrm{d} \text { vs } \\
\text { placebo }\end{array}$ & 2 weeks & $\begin{array}{c}\text { Pain (VAS, } \\
\text { NRS McGill } \\
\text { questionnaire) } \\
\text { Sleep (Leeds } \\
\text { Sleep evaluation } \\
\text { questionnaire) } \\
\text { HRQL }\end{array}$ & $\begin{array}{l}\text { Improved sleep \& } \\
\text { significantly lower } \\
\text { pain intensity }\end{array}$ & $\begin{array}{l}\text { No withdrawals } \\
\text { drowsiness }\end{array}$ \\
\hline 14 & $\begin{array}{l}\text { Nurmikko } \\
\text { et al.[10] }\end{array}$ & Crossover & Neuropathic pain & 63 & 62 & $\begin{array}{c}\text { Nabixmols: } \\
\text { THC (30mg)/ } \\
\text { Cannibidol } \\
(27.5 \mathrm{mg}) \\
\text { oromucosal spray } \\
\text { vs Placebo }\end{array}$ & 5 weeks & $\begin{array}{l}\text { Pain (NRS) } \\
\text { Sleep PGIC }\end{array}$ & $\begin{array}{l}\text { Significant decrease } \\
\text { in pain \& improved } \\
\text { sleep }\end{array}$ & $18 \%$ dropped out \\
\hline 15 & $\begin{array}{c}\text { Berman et } \\
\text { al.[30] }\end{array}$ & Crossover & Neuropathic pain & 48 & 48 & $\begin{array}{c}\text { Nabixmols: } \\
\text { THC (129.6mg)/ } \\
\text { Cannibidol } \\
(120 \mathrm{mg}) \\
\text { oromucosal spray } \\
\text { vs Placebo }\end{array}$ & 2 weeks & $\begin{array}{l}\text { Pain Sleep } \\
\text { SF-MPQ PDI }\end{array}$ & $\begin{array}{l}\text { Significant decrease } \\
\text { in pain and sleep } \\
\text { improvement }\end{array}$ & $\begin{array}{l}3 \text { withdrawals for } \\
\text { nausea vomiting } \\
\text { fainting Intoxication } \\
\text { type reactions, being } \\
\text { mild to moderate in } \\
\text { severity and resolving } \\
\text { spontaneously }\end{array}$ \\
\hline 16 & $\begin{array}{l}\text { Karst et } \\
\text { al.[17] }\end{array}$ & Crossover & Neuropathic pain & 21 & 21 & $\begin{array}{c}\text { CT-3 }(4 \times 10 \mathrm{mg}) \\
\text { vs placebo }\end{array}$ & 7 days & Pain (VAS) & $\begin{array}{l}\text { Reduction in pain } \\
\text { scores }\end{array}$ & $\begin{array}{l}1 \text { withdrawal Dry } \\
\text { mouth \& Tiredness }\end{array}$ \\
\hline 17 & $\begin{array}{l}\text { Wade et } \\
\text { al.[29] }\end{array}$ & Crossover & Neuropathic pain & 20 & 20 & $\begin{array}{l}\text { THC }(2.5 \mathrm{mg}) \\
\text { vs CBD vs } \\
\text { Nabixmols }(120 \\
\text { mg) vs placebo }\end{array}$ & 10 weeks & Pain (VAS) & $\begin{array}{l}\text { THC \& CBD were } \\
\text { superior to placebo }\end{array}$ & $\begin{array}{c}3 \text { dropped out due to } \\
\text { hypotension }\end{array}$ \\
\hline 18 & $\begin{array}{l}\text { Rog et } \\
\text { al.[28] }\end{array}$ & Parallel & Neuropathic pain & 66 & 66 & $\begin{array}{l}\text { Nabixmols vs } \\
\text { placebo }\end{array}$ & 5 weeks & $\begin{array}{l}\text { Pain (NRS) } \\
\text { Sleep HADS } \\
\text { PGIC }\end{array}$ & $\begin{array}{l}\text { Significant decrease } \\
\text { in pain intensity and } \\
\text { improved sleep }\end{array}$ & 2 withdrawals \\
\hline 19 & $\begin{array}{l}\text { Frank et } \\
\text { al.[16] }\end{array}$ & Crossover & Neuropathic pain & 48 & 48 & $\begin{array}{l}\text { Dihydrocodeine } \\
240 \mathrm{mg} \text { vs } \\
\text { nabilone } 2 \mathrm{mg} \\
\text { orally }\end{array}$ & 12 weeks & $\begin{array}{l}\text { Pain (VAS) } \\
\text { Depression } \\
\text { (Hamilton) } \\
\text { SF-36 }\end{array}$ & $\begin{array}{l}\text { Dihydrocodeine } \\
\text { provided better pain } \\
\text { relief }\end{array}$ & $\begin{array}{c}33 \text { withdrawals } \\
\text { Tiredness, sickness, } \\
\text { tingling and } \\
\text { nightmares }\end{array}$ \\
\hline \multirow[b]{2}{*}{20} & \multirow{2}{*}{$\begin{array}{l}\text { Toth et } \\
\text { al.[24] }\end{array}$} & \multirow[b]{2}{*}{ Parallel } & \multirow{2}{*}{$\begin{array}{l}\text { Diabetic } \\
\text { neuropathy }\end{array}$} & \multirow[b]{2}{*}{25} & \multirow[b]{2}{*}{26} & \multirow{2}{*}{$\begin{array}{l}\text { Nabilone vs } \\
\text { placebo }\end{array}$} & \multirow[b]{2}{*}{5 weeks } & $\begin{array}{c}\text { Pain (NRS) } \\
\text { Function (BPI) } \\
\text { QOL (EQ-5D) }\end{array}$ & \multirow{2}{*}{$\begin{array}{c}\text { Nabilone was } \\
\text { statistically more } \\
\text { effective in reducing } \\
\text { pain intensity, } \\
\text { improvement in sleep } \\
\text { and anxiety }\end{array}$} & \multirow[b]{2}{*}{ No withdrawals } \\
\hline & & & & & & & & $\begin{array}{l}\text { Satisfaction } \\
\text { (PTSS) Mood } \\
\text { (HADS) Sleep } \\
\text { (MOSS) }\end{array}$ & & \\
\hline 21 & $\begin{array}{l}\text { Serpell et } \\
\text { al.[33] }\end{array}$ & Parallel & $\begin{array}{l}\text { Peripheral } \\
\text { neuropathy }\end{array}$ & 128 & 118 & $\begin{array}{c}\text { Oromucosal } \\
\text { cannabis vs } \\
\text { placebo }\end{array}$ & 15 weeks & $\begin{array}{c}\text { Pain (NRS) } \\
\text { Function (BPI) } \\
\text { QOL (EQ-5D) } \\
\text { Satisfaction } \\
\text { (PTSS) } \\
\text { Mood(HADS) } \\
\text { Sleep } \\
\text { PGIC }\end{array}$ & $\begin{array}{l}\text { Statistically } \\
\text { significant } \\
\text { reduction in pain } \\
\text { \& improvements in } \\
\text { sleep and GIC }\end{array}$ & $\begin{array}{l}73 \text { withdrawals due } \\
\text { to loss of efficacy and } \\
\text { side effects }\end{array}$ \\
\hline
\end{tabular}

Table 2: Summary of efficacy in single studies.

challenges, faced in pain research. In this review, only 4 trials stated the exact definition for the pain condition they investigated together with the guidelines criteria conventionally used for patients' diagnosis. In contrast, other trials used telephone or surveys to recruit patients and were only satisfied by patients' statements on their diagnosis without further investigations to confirm the diagnosis [31]. Three trials recruited patients with different types of CP condition. Then, these trials collectively analysed the results for all patients together regardless of their condition, while distinctions should have been made. Moreover, the duration of $\mathrm{CP}$ condition was considered neither as selective criteria nor as a confounding factor that might have affected the effectiveness of the drug under investigation [32].

For many CP conditions, many patients used either over the counter medications without ever seeking medical assistance or getting documented in any health records or used concomitant drugs and on various doses or other non-pharmacological approaches (e.g., physical therapy). However, none of the RCTs included this criterion as a selective one. Although such criterion provided the opportunity to evaluate potential pharmacodynamics interactions, nevertheless, it was not considered as a confounding factor in the 21 trials.

Patients who had past history of smoking cannabis were not excluded from the trials, which had added to the heterogeneity to the trials. Although these subjects represent typical clinical CP patients, a more homogeneous sample may have had a different outcome [33].

The sample size used in all selected 21 trials ranged from 13 to 128 with relatively small sample sizes; therefore, all findings regarding the efficacy of cannabinoids were questionable.

\section{Intervention}

$\mathrm{CP}$ is a chronic disease, expected to persist for lifetime and treatment is expected to be utilized for long periods of time. To assess the efficacy 


\begin{tabular}{|c|c|c|c|c|c|c|c|c|c|c|c|c|c|c|c|c|c|c|c|c|c|}
\hline & & 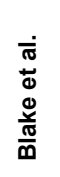 & 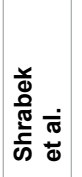 & 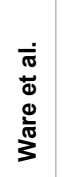 & 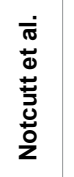 & 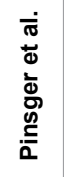 & 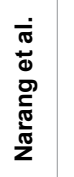 & 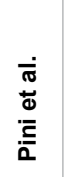 & 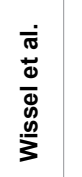 & 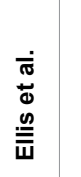 & 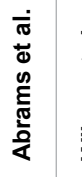 & 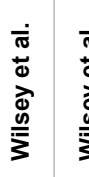 & 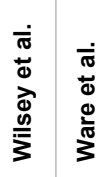 & 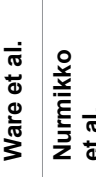 & 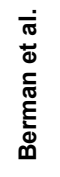 & 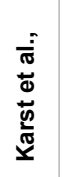 & 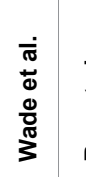 & 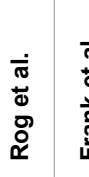 & 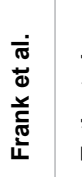 & 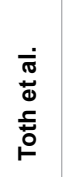 & 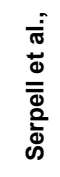 \\
\hline \multirow{2}{*}{ Randomization } & $\begin{array}{l}\text { Random } \\
\text { sequence }\end{array}$ & $\vee$ & $\vee$ & $v$ & $v$ & $v$ & $\checkmark$ & $\checkmark$ & $v$ & $\checkmark$ & $\vee$ & $v v$ & $v \vee$ & $v v$ & $\vee$ & $\checkmark$ & $\checkmark$ & $\vee$ & $\vee$ & $v$ & $v$ \\
\hline & Allocation & $\checkmark$ & $\checkmark$ & $?$ & $?$ & $?$ & $v$ & $?$ & $?$ & $?$ & $?$ & $v v$ & $\vee ?$ & $? \vee$ & $v$ & $\checkmark$ & $?$ & ? & ? & $?$ & $?$ \\
\hline \multirow{2}{*}{ Blinding } & $\begin{array}{c}\text { Blinding } \\
\text { participants }\end{array}$ & $\boldsymbol{\otimes}$ & $\boldsymbol{\otimes}$ & $\boldsymbol{x}$ & $\checkmark$ & $\boldsymbol{x}$ & $\checkmark$ & $\otimes$ & $v$ & $\checkmark$ & $\vee$ & $\checkmark 8$ & $\boldsymbol{x} v$ & 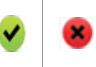 & $\vee$ & $\boldsymbol{x}$ & $\vee$ & $\checkmark$ & $\checkmark$ & $v$ & $v$ \\
\hline & $\begin{array}{l}\text { Blinding } \\
\text { researchers }\end{array}$ & $\checkmark$ & $\vee$ & $v$ & $v$ & $v$ & $\checkmark$ & $\vee$ & $v$ & $\checkmark$ & $\vee$ & $v \vee$ & $v \vee$ & $v \vee$ & $\checkmark$ & $v$ & 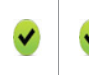 & $\vee$ & $\vee$ & $v$ & $v$ \\
\hline Disposition & $\begin{array}{l}\text { Withdrawals } \\
\text { \& Dropouts }\end{array}$ & $\boldsymbol{\otimes}$ & $v$ & $v$ & $>$ & $\boldsymbol{x}$ & $\vee$ & $\checkmark$ & $v$ & $v$ & $\vee$ & $v \vee$ & $v v$ & $v v$ & $\vee$ & $v$ & $v(1$ & $v \quad v$ & $\vee$ & $v$ & $v$ \\
\hline
\end{tabular}

of a drug, there is a need for trials of longer duration to test for longterm efficacy as well as any potential chance for side-effects or abuse. Nevertheless, the duration in all selected trials ranged from 5 days to 6 weeks. Moreover, the period varied across all trials without any standardized time interval determined to assess effectiveness. 15 of 21 trials compared a cannabinoid to a placebo 3 compared cannabinoids to another drug and a placebo [34]. While 3 trials compared cannabinoid to other drugs without using a placebo or control group. A concern in the trials without placebo control is that it is impossible to be certain that any reported significant improvements are due to the drug under investigation since many trials report placebo response ranging from 20 to $70 \%$. Therefore, the reported results in the 3 trials could not be reliable unless there was a placebo control. 6 trials assessed the efficacy of smoked cannabis. In these trials, the study nurse explained the method of drug administration from an adjacent room and participants were then left to smoke without accurate calculation to the dose of the administered drug or close monitoring for the compliance with these instructions.

Moreover, dose titration protocols varied according to individual differences in sensitivity to the analgesic and adverse effects of cannabis; each participant titrated to the dose affording the maximum pain relief with the minimum adverse effects. The method of administration also varied from one trial to another; administration routes for cannabinoids included vaporization and mucosal sprays. Together, the various doses and ways of administration made the comparative analysis of cannabinoid efficacy very challenging.

\section{Randomization}

It was reported in all 21 trials suggesting a low risk of bias (Table 3). Nevertheless, the method used to generate the sequence of randomization or allocation was rarely reported or not well described in any of the trials.

\section{Blinding}

All trials were designed as double blinded trials. Blinding of both participants and observers was documented in all studies. However, the effectiveness of blinding was doubtful. At the end of the study, the subjects were asked to guess which group they had been designated for (experimental or control) as a means of estimating the success of blinding. In 8 trials, participants were able to correctly identify their group, suggesting ineffective participant blinding.

\section{Results}

In all 21 trials, participants were randomly assigned to the study groups and each group received intended treatment. Consequently, they were analysed for the primary (and secondary) outcome(s). Taken together, the 21 trials demonstrated a modest analgesic effect in pain as a primary outcome. In addition, 9 trials reported improvement in sleep. Drug-related adverse effects were generally described as well tolerated and most commonly included sedation, dizziness, dry mouth, nausea, and vomiting. Withdrawals potentially related to drug-related side effects was reported, however, it was minimal and did not seem to be associated with bias.

\section{Outcomes}

Conventionally in acute pain research, the response to a treatment is assessed by how much it reduces pain intensity; nevertheless, CP patients' experience usually entails a range of serious implications, of which pain intensity is but one feature.

In all selected trials, the predominant outcome to be assessed, and therefore, the primary efficacy measure was not functional, emotional, or societal impact but pain intensity. All trials used various pain intensity scales as their primary outcome to assess the efficacy of cannabinoid on $\mathrm{CP}$, ignoring other relevant outcomes to the condition under investigation. As a result, when all but one study, found a significant decrease in pain intensity related to cannabinoids administration, they concluded that cannabinoids were effective in managing CP compared to controls or other drugs. Hence, the determination of efficacy was mainly based on somewhat arbitrary rating scales that might not be clinically meaningful.

13 studies used other variables as secondary outcomes such as sleep, mood, anxiety, and depression, physical function and quality of life, the results for these outcomes were not clearly reported or nearly absent. Only 7 trials reported improvements in sleep, 5 trials showed improvements in quality of life. While 3 trials reported improvements in all measured outcomes. The variability in outcome measures, also, made it very difficult to evaluate and to conclude the real efficacy of cannabinoids. 


\section{Side effects and withdrawals}

Patients' side effects were reported in all trials except for two neurocognitive adverse effects were reported as the most common (e.g., headache, dry mouth dysphoria, dizziness, and sedation). All reported side effects were generally described as well tolerated, and not leading to withdrawals. It is worthy to note, however, that as all selected RCTs were short-term trials and all used relatively small doses that could have masked potential severe side effects. Given the psycho active properties of cannabinoids, several side-effects could be expected, including overdose, abuse, dependence, and even addiction. Additionally, as all drugs, cannabinoids can pose a toxic effect. However, end-organ failure secondary to medication was not assessed.

Withdrawals potentially related to drug-related side effects was reported, however, it was minimal and did not seem to be associated with bias. Although, these side-effects constituted from 3-18\% of all participants, nevertheless, the majority of trials showed that lack or loss of effectiveness was the main reason for withdrawals.

\section{Discussion}

In reviewing RCTs investigating the efficacy of cannabinoids in managing $\mathrm{CP}$ different conditions, only 21 trials were found. There is a remarkable paucity in RCTs given the dramatic increase in cannabinoids use among CP patients. RCTs employed different methods and investigated various CP conditions. Despite discrepancies, all but one trial concluded that cannabinoids are effective in managing $\mathrm{CP}$ conditions, especially, neuropathic pain.

\section{Implications for theory, research and clinical practice}

\section{Theory}

In 1960, Ronald Melzak and Patrick Wall developed the gate control theory. Since then, the gate theory has been helping us understanding many of the complex pain conditions that we witness in practice. The theory shows that the experience of pain depends on a complex interplay of multiple factors, and pain sensation is the net result of the interactions between them. This new perception urges the research community to assess all factors affected by pain when assessing the efficacy of treatments. Eventually, there have been few tentative steps in this direction. Nevertheless, while research in cannabinoids is much better in this respect compared to opioids' trials in CP; this new perspective is yet to be effectively translated to research methodologies.

\section{Research}

Cannabinoids are now increasingly used, more without prescription. Remarkably, previous systematic reviews have concluded that cannabinoids are effective in treating $\mathrm{CP}$. What is surprising is that they were able to reach a conclusion, although the quality of RCTs were lacking. It seems that researchers are pressured to agree on relatively weak evidence just to follow the flow of use rather than waiting for more rigorous studies. It's, therefore, critical to efficiently assess the effectiveness of Cannabinoids. So far RCTs, conducted to assess the effectiveness of cannabinoids, have been associated with many limitations. The ambiguity of $\mathrm{CP}$ conditions, the discrepancies and variability in the methodology employed by RCTs were all confounding factors. The inclusion criteria of participants included in these trials were overwhelmingly different. Therefore, conclusions of these trials might be inappropriate to generalize or apply in clinical practice. As a result, this review cannot reach certainty regarding potential benefits as well as the serious side effects that may be associated with the use of cannabinoids for CP conditions on long-term basis. Nevertheless, "the absence of evidence of effect is not the same as the evidence of absence of effect"; i.e. not because there is no consistent evidence for the efficacy of cannabinoids in CP, this does not mean that they don't have any.

Researchers have the responsibility to provide evidence-based guidance on this important potential treatment. Additional trials need to be conducted in the future to further assess the risks and benefits of this potential drug, and to determine the most effective dose and the best method of administration. Future trials to determine the effectiveness of Cannabinoids for CP patients should be comprehensive focusing on risk-benefits assessments. Demographics of any RCT should be reported in details and in a more selective manner so that clinicians can determine how relevant the results to their patients. Researchers need to use a uniform method, beside the recommended IMMPACT outcomes to ensure standardization of pain research. The results of RCTs should be meaningful or clinically relevant to the same type of patients that resemble the participants that were studied.

\section{Clinical practice}

Owing to increasing public pressure, the Canadian government has set some regulations whereby cannabinoids can be obtained legally by prescription. However, clinicians have expressed concerns about the associated side effects.

Unfortunately, the absence of high-quality trials does not allow for consistent guidelines or recommendations for clinical practice use. The low quantity and quality of data available cannot provide real evidence to confirm efficacy or harm for routine clinical use. We can only say that Cannabinoids can be a potential treatment for CP; however, the same rules/ precautions applied to opioids should be also employed with the Cannabinoids prescription. I think the decision on using Cannabinoids will remain as a one that is mainly based on the clinician's experience as well as the patient's desire until evidence-based reports become available.

\section{Conclusions}

In conclusion, this review of 21 trials demonstrates that cannabinoids can be a potential treatment option for CP. Nevertheless, it was really challenging to conclude a definitive effectiveness for the cannabinoids since there were no standardized objective outcome tools to assess effectiveness in pain trials and because of the variable methods used.

Unfortunately, this paucity in research persists despite the dramatic increase in Cannabinoids use in many different forms between $\mathrm{CP}$ patients.

\section{References}

1. Lynch ME, Campbell $F$ (2011) Cannabinoids for treatment of chronic noncancer pain: A systematic review of randomized trials. $\mathrm{Br} \mathrm{J}$ Clin Pharmacol 72: 735-744.

2. Blake D, Robson P, Ho M, Jubb RW, McCabe CS (2006) Preliminary assessment of the efficacy, tolerability and safety of a cannabis-based medicine (Sativex) in the treatment of pain caused by rheumatoid arthritis. Rheumatology 45: 50-52.

3. Deshpande A, Mailis-Gagnon A, Zoheiry N, Lakha SF (2015) Efficacy and adverse effects of medical marijuana for chronic noncancer pain Systematic review of randomized controlled trials. Canadian Family Physician 61: e372-e381.

4. Pini LA, Guerzoni S, Cainazzo MM, Ferrari A, Sarchielli P, et al. (2012) Nabilone for the treatment of medication overuse headache: results of a preliminary double-blind, active-controlled, randomized trial. J Headache Pain 13: 677-684

5. Skrabek RQ, Galimova L, Ethans K, Perry D (2008) Nabilone for the treatment of pain in fibromyalgia. The Journal of Pain 9: 164-173.

6. Ware MA, Wang T, Shapiro S, Robinson A, Ducruet T, et al. (2010) Smoked cannabis for chronic neuropathic pain: A randomized controlled trial. CMAJ 182: E694-E701. 
Citation: Hassan S (2018) Cannabinoids for the Treatment of Chronic Pain: A Critical Review of Randomized Controlled Trials. J Pain Manage Med 4: 131.

7. Wilsey B, Marcotte T, Tsodikov A, Millman J, Bentley H, et al. (2008) A randomized, placebo-controlled, crossover trial of cannabis cigarettes in neuropathic pain. J Pain 9: 506-521.

8. Wissel J, Haydn T, Müller J, Brenneis C, Berger, et al. (2006) Low dose treatment with the synthetic cannabinoid nabilone significantly reduces spasticity-related pain: A double-blind placebo-controlled cross-over trial. Journal of neurology, 253: 1337-1341.

9. Klein KB (1988) Controlled treatment trials in the irritable bowel syndrome: A critique. Gastroenterology, 95: 232-241.

10. Nurmikko TJ, Serpell MG, Hoggart B, Toomey PJ, Morlion BJ, et al. (2007) Sativex successfully treats neuropathic pain characterised by allodynia: $A$ randomised, double-blind, placebo-controlled clinical trial. Pain 133: 210-220.

11. Schulz KF, Altman DG, Moher D (2010) CONSORT 2010 statement: Updated guidelines for reporting parallel group randomised trials. BMJ 340: c332.

12. Schopflocher D, Taenzer P, Jovey R (2011) The prevalence of chronic pain in Canada. Pain Res Manag 16: 445-450.

13. Fitzcharles MA, Baerwald C, Ablin J, Häuser W (2016) Efficacy, tolerability and safety of cannabinoids in chronic pain associated with rheumatic diseases (fibromyalgia syndrome, back pain, osteoarthritis, rheumatoid arthritis): A systematic review of randomized controlled trials. Schmerz 30: 47-61.

14. Landmark T, Romundstad P, Dale O, Borchgrevink PC, Vatten L, et al. (2013) Chronic pain: One year prevalence and associated characteristics (the HUNT pain study). Scandinavian Journal of Painn 4: 182-187.

15. Abrams DI, Jay CA, Shade SB, Vizoso H, Reda H, et al. (2007) Cannabis in painful HIV-associated sensory neuropathy: A randomized placebo-controlled trial. Neurology, 68: 515-521.

16. Frank B, Serpell MG, Hughes J, Matthews JN, Kapur D (2008) Comparison of analgesic effects and patient tolerability of nabilone and dihydrocodeine for chronic neuropathic pain: Randomised, crossover, double blind study. Bmj 336: 199-201.

17. Karst M, Salim K, Burstein S, Conrad I, Hoy L, et al. (2003) Analgesic effect of the synthetic cannabinoid CT-3 on chronic neuropathic pain: A randomized controlled trial. Jama, 290: 1757-1762.

18. Lynch ME, Craig KD, Peng PW (2011) The challenge of pain: A multidimensiona phenomenon. Clinical pain manage

19. Wilsey B, Marcotte T, Deutsch R, Gouaux B, Sakai S, et al. (2013) Low-dose vaporized cannabis significantly improves neuropathic pain. J Pain 14: 136148.

20. Serpell M, Ratcliffe S, Hovorka J, Schofield M, Taylor L, et al. (2014) A doubleblind, randomized, placebo-controlled, parallel group study of THC/CBD spray in peripheral neuropathic pain treatment. Eur J Pain 18: 999-1012.
21. Toth C, Mawani S, Brady S, Chan C, Liu C, et al. (2012) An enriched-enrolment randomized withdrawal, flexible-dose, double-blind, placebo-controlled, paralle assignment efficacy study of nabilone as adjuvant in the treatment of diabetic peripheral neuropathic pain. Pain 153: 2073-2082.

22. Lynch ME (2011) The need for a Canadian pain strategy. Pain Res Manag 16 $77-80$.

23. Aggarwal SK (2013) Cannabinergic pain medicine: A concise clinical prime and survey of randomized-controlled trial results. Clin J Pain 29: 162-171.

24. Pinsger M, Schimetta W, Volc D, Hiermann E, Riederer F, et al. (2006) [Benefits of an add-on treatment with the synthetic cannabinomimetic nabilone on patients with chronic pain--a randomized controlled trial]. Wiener klinische Wochenschr 118: 327-335.

25. Rog DJ, Nurmikko TJ, Friede T, Young CA (2005) Randomized, controlled tria of cannabis-based medicine in central pain in multiple sclerosis. Neurology, 65: 812-819.

26. Wade DT, Robson P, House H, Makela, P, Aram J (2003) A preliminary controlled study to determine whether whole-plant cannabis extracts can improve intractable neurogenic symptoms. Clin Rehabil.17: 21-29.

27. Berman JS, Symonds C, Birch R (2004) Efficacy of two cannabis based medicinal extracts for relief of central neuropathic pain from brachial plexus avulsion: Results of a randomised controlled trial. Pain 112: 299-306.

28. Ware MA, Fitzcharles MA, Joseph L, Shir Y (2010) The effects of nabilone on sleep in fibromyalgia: Results of a randomized controlled trial. Anesth Analg 110: 604-610.

29. Ellis RJ, Toperoff W, Vaida F, Van Den Brande G, Gonzales J, et al. (2009) Smoked medicinal cannabis for neuropathic pain in HIV: A randomized crossover clinical trial. Neuropsychopharmacology, 34: 672-680.

30. Hill KP (2015) Medical marijuana for treatment of chronic pain and othe medical and psychiatric problems: A clinical review. Jama 313: 2474-2483.

31. Lynch ME, Ware MA (2015) Cannabinoids for the treatment of chronic noncancer pain: An updated systematic review of randomized controlled trials. J Neuroimmune Pharmacol 10: 293-301.

32. Melzack R (1996) Gate control theory: On the evolution of pain concepts. Pain forum 5:128-138.

33. Narang S, Gibson D, Wasan AD, Ross EL, Michna E, et al. (2008) Efficacy of dronabinol as an adjuvant treatment for chronic pain patients on opioid therapy. J Pain 9: 254-264.

34. Notcutt W, Price M, Miller R, Newport S, Phillips C, et al. (2004). Initial experiences with medicinal extracts of cannabis for chronic pain: Results from 34 ' $\mathrm{N}$ of 1'studies. Anaesthesia 59: 440-452. 